\title{
Footprint Modeling of Cache Associativity and Granularity
}

\author{
Hao Luo* \\ Google Inc. \\ Mountain View, California, USA \\ haoluo@google.com \\ Pengcheng $\mathrm{Li}^{*}$ \\ Google Inc. \\ Mountain View, California, USA \\ pengchengli@google.com
}

\author{
Guoyang Chen ${ }^{\dagger}$ \\ Alibaba Group US Inc. \\ Sunnyvale, California, USA \\ g.chen@alibaba-inc.com \\ Chen Ding \\ University of Rochester \\ Rochester, New York, USA \\ cding@cs.rochester.edu
}

\author{
Fangzhou Liu \\ University of Rochester \\ Rochester, New York \\ fliu14@cs.rochester.edu \\ Xipeng Shen \\ North Carolina State University \\ Raleigh, North Carolina, USA \\ xshen5@ncsu.edu
}

\begin{abstract}
Two common techniques in efficient caching are associativity and sub-block granularity. This short paper presents a parameterized and composable model for each of the two techniques. It shows how the new models are more general, accurate or efficient than previous modeling solutions in either technique, and how they can be used together to model the cache implemented with both techniques, i.e. sub-block set associative cache.
\end{abstract}

CCS Concepts Computing methodologies $\rightarrow$ Modeling methodologies;

Keywords Partial Footprint, Mapped Footprint, Dual-grained Footprint, Joint Modeling

\section{ACM Reference Format:}

Hao Luo, Guoyang Chen, Fangzhou Liu, Pengcheng Li, Chen Ding, and Xipeng Shen. 2018. Footprint Modeling of Cache Associativity and Granularity. In The International Symposium on Memory Systems (MEMSYS), October 1-4, 2018, Old Town Alexandria, VA, USA. ACM, New York, NY, USA, 11 pages. https://doi.org/10.1145/ 3240302.3240419

\footnotetext{
${ }^{*}$ This work was done when the author was a graduate student at University of Rochester.

${ }^{\dagger}$ This work was done when the author was a graduate student at North Carolina State University.
}

Permission to make digital or hard copies of all or part of this work for personal or classroom use is granted without fee provided that copies are not made or distributed for profit or commercial advantage and that copies bear this notice and the full citation on the first page. Copyrights for components of this work owned by others than the author(s) must be honored. Abstracting with credit is permitted. To copy otherwise, or republish, to post on servers or to redistribute to lists, requires prior specific permission and/or a fee. Request permissions from permissions@acm.org. MEMSYS, October 1-4, 2018, Old Town Alexandria, VA, USA

(C) 2018 Copyright held by the owner/author(s). Publication rights licensed to ACM.

ACM ISBN 978-1-4503-6475-1/18/10 ..\$15.00

https://doi.org/10.1145/3240302.3240419

\section{Introduction}

Memory performance is essential to parallel performance. This paper is concerned with the two common caching techniques: associativity and sub-block granularity.

We take the approach of model-driven optimization. Modeling is fast. It samples a program execution at the beginning, calculates the performance of all possible implementations, and picks the best one for the rest of the execution. The optimization is based on performance models rather than exhaustive testing, an approach which can help selecting the best configurations through executing or simulating all possible implementations.

In this paper, we solve the following three problems:

- Nonuniform data locality. A program uses multiple arrays, which have different access frequency and locality.

- Uneven address mapping. In set-associative cache, data addresses may be mapped unevenly to cache sets. In addition, the actual mapping can be changed for the L1 data cache by configuring it into a different capacity and hence a different number of cache sets.

- Sub-block cache. In sub-block cache, or sector cache ${ }^{1}$, the full cache block was split into smaller units named "sub-blocks" or "sectors" [5], and the cache fetches a new sub-block only each time there is a cache miss. Hence, the granularity for fetching and managing data is different.

These three problems are different forms of heterogeneity: non-uniform data locality in software, sub-block cache in hardware, and uneven mapping between software and hardware. In this paper, we present three models, each solving one problem. We then combine the models to cover the interaction across all three types of heterogeneity.

The combined analysis helps a programmer to gain insight. For example, she can dissect and quantify the performance of a single array as she changes the peer arrays in cache

\footnotetext{
${ }^{1}$ In the rest of the paper, we use the term sub-block cache and sector cache interchangeably.
} 
and the size, associativity, or type of cache. All models use a single measurement, maximizing the speed and consistency of the multi-factor analysis. With this benefit, our models help cache designers as well to gain efficiency, as they have to try many parameters, like the cache size, associativity, for best cache performance. With our models, they can quantify the cache performance (i.e. cache miss ratio) for all possible configurations and select the best one without repeated testing.

Our new models overcome important limitations of previous techniques. Testing and simulation have a high cost, which restricts the space of exploration, e.g. a few but not all cache sizes. Concurrent reuse distance can evaluate all cache sizes [23] but for a fixed task or data group, not all subgroups. The working-set models are composable but ignore the effect of non-uniform address mapping in set-associative cache, whose effect is more pronounced on GPUs than on CPUs. Nugteren et al. modeled set-associative cache of any size on GPUs [16] but for a fixed number of cache sets (and a fixed task/array group). The new methods remove these limitations individually and more importantly, permit their combination and joint optimization.

The main contributions of the paper are as follows:

- two techniques called time-preserving trace decomposition and the partial footprint to characterize the non-uniform data locality (Section 2.2),

- the mapped footprint to model the effect of the cache size, the number of cache sets, and the associativity of the set-associative cache (Section 2.3),

- the dual-grained footprint to model the sub-block cache (Section 2.4),

- the composition of the above models and the correctness theorem (Section 2.5).

- the evaluation of the individual and combined analysis on 13 GPU benchmarks and comparison with three existing techniques. (Section 3),

In this work, we model the locality effect, i.e. the misses in memory accesses, not execution time (cycles). Locality models show the relation between program organization and hardware utilization, e.g. cache occupancy. Yet they are machine independent and therefore enable program optimization across different memory hierarchies.

\section{Composable Data-centric Models}

This section first introduces the background theory and then presents the three new models.

\subsection{Background: Footprint}

Xiang et al. gave the higher-order theory of locality (HOTL) which defines a set of metrics and uses them to compute the miss ratio in shared cache [25]. The most important metric is footprint.
In an execution trace, each time window is represented by $(t, x)$, where $t$ is the end position and $x$ the window length. The number of distinct elements in the window is the working-set size $\omega(t, x)$ [8]. For each $x, f p(x)$ is the average working-set size of all windows of length $x$, i.e. the total working-set size divided by the number of length- $x$ windows as shown by the following equation:

$$
f p(x)=\frac{1}{n-x+1} \sum_{t=x}^{n} \omega(t, x)
$$

The parameter $x$ an integer time scale $0 \leq x \leq n$, where $n$ is the trace length.

For fully-associative LRU cache, the miss ratio $m r(c)$ is the (discrete) derivative of the footprint function. The precise formula has two calculations as follows. Given cache size $c$, it first finds the time scale $x$ and then takes the derivative at $x$.

$$
m r(c)=f p(x+1)-f p(x) \text { where } c=f p(x)
$$

In this paper, we refer to it as the HOTL conversion and use a more compact representation based on the Leibniz's notation, where the two calculations are in the same equation separated by the vertical bar.

$$
m r(c)=\left.\frac{\mathrm{d}}{\mathrm{d} x} f p(x)\right|_{f p(x)=c}
$$

As an example illustration, the following table shows a simple access trace, its footprint, and the miss ratios computed using the HOTL conversion.

Table 1. Example Footprint and HOTL Conversion

(a) An access trace

access trace: $a$ b c

(b) Footprint

\begin{tabular}{c|l|l|l|l} 
time scale $x$ & 0 & 1 & 2 & 3 \\
\hline$f p(x)$ & 0 & 1 & 2 & 3
\end{tabular}

(c) Miss ratios

\begin{tabular}{c|c|c|c} 
cache size $c$ & 0 & 1 & 2 \\
\hline$m r(c)$ & $100 \%$ & $100 \%$ & $100 \%$
\end{tabular}

The HOTL theory includes efficient footprint measurement, concavity of the footprint (hence monotonicity of the miss ratio), and the correctness condition [24, 25]. This paper builds on these results and will use the HOTL conversion extensively. 
HOTL Optimization and Limitations The HOTL theory has been shown effective for optimizing program symbiosis [21, 25], cache partition-sharing for hardware cache [2], and memory allocation for software cache [11]. However, HOTL still has at least four limitations. First, footprint optimization targeted tasks, not data. It cannot show the which data to use cache. Second, HOTL assumes uniform or average interleaving between accesses of independent programs, but in systems like GPU, data can be interleaved arbitrarily. In this case, we cannot use footprint to model the cache performance. Third, HOTL does not model uneven address mapping. Finally, HOTL does not handle the sub-block cache. Next we describe three new models to overcome these limitations: the partial footprint for the first two and the mapped and dual-granularity footprints for the latter two.

\subsection{Modeling Nonuniform Data Locality}

In this section, we present two techniques: time-preserving decomposition to account for non-uniform interleaving, and partial footprint for uneven locality. Using them together, we can derive the miss ratio of all array groups for all cache sizes.

\subsubsection{Time-preserving Decomposition}

Let $\Omega=\left\{d_{i}\right\}$ be the set of arrays used by the program. Each member $d_{i}$ may be an array of any dimension or size. The only assumption we make is that different $d_{i}$ s do not overlap in memory.

Assume that the execution of this program can be collected as an interleaved trace of memory addresses. Time-preserving decomposition splits it into a set of traces, one for each $d_{i}$. In each $d_{i}$ trace, all accesses to $d_{i}$ are kept, and others are replaced by placeholders. This decomposition is done in a single pass over the interleaved trace.

We define a general concept called a time preserving (TP) trace, which is a copy of the original trace with 0 or more accesses replaced with placeholders. A TP trace is time preserving because its accesses have the same access time as in the original trace. In fact, the original trace is a TP trace. All TP traces have the length of the original trace $n$. In timepreserving decomposition, each $d_{i}$ trace is a TP trace.

For example, consider the trace of accesses to three variables: $a b c$ abc abc.... The decomposition generates three TP traces, one for each array: $a_{-} a_{-} a_{-} \ldots, b_{-} b_{-} b_{-} b_{-} \ldots$ and __ _ _ _ _ _ c..., where each TP trace contains accesses to a single variable, and all other accesses are turned into placeholders “_”.

\subsubsection{Partial Footprint}

The locality of a TP trace is measured by its partial footprint. The definition is identical to that of footprint (based on the whole trace, in Section 2.1), with just one change: a TP trace has placeholders, and they do not add to any working set. Let $s_{i}$ be a TP trace, its partial footprint is defined by

$$
p f p(x)=\frac{1}{n-x+1} \sum_{j=x}^{n} \omega\left(s_{i}, j, x\right)
$$

As a TP trace generalizes a trace by allowing placeholders, the partial footprint generalizes the footprint as the measure of locality. The original trace is a TP trace, and the footprint defined in HOTL is now a special case of partial footprint. In the rest of the paper, we use the terms footprint and partial footprint interchangeably.

We define the locality of an array $d_{i}$ as the partial footprint of its TP trace, denote as $p f p\left(d_{i}, x\right)$. In the $d_{i}$ TP trace, in each window, the number of distinct elements is the $d_{i}$ working set. The partial footprint $p f p\left(d_{i}, x\right)$ is the average size of the $d_{i}$ working set.

Consider the TP trace $a_{-} a_{-} a_{-} a_{\ldots} \ldots$. The partial footprint is $p f p(a, 1)=\frac{1}{3}$, because two out of every three accesses are placeholders. If a variable $z$ is never accessed, its TP trace has only placeholders, and its partial footprint is $p f p(z, x)=0$ for all $0 \leq x \leq n$.

The locality of a group of arrays can be defined similarly. We first obtain the TP trace with only accesses to the array group. The group locality is then the partial footprint of this TP trace. Fortunately, it is not necessary to build a TP trace and measure - the group locality can be "composed" from individual locality.

\subsubsection{Locality Composition}

The partial footprint supports composable analysis. Composable analysis has two steps. In the first step, we measure the partial footprint of each array. Then we do not need TP traces any more. In the second step, we can obtains the aggregate footprint for any group of arrays through calculation rather than measurement.

The partial footprint is composable, which means that the group footprint is the sum of the footprint of its group members. For any group $\mathcal{D}=\left\{d_{i}\right\}$ and all $0 \leq x \leq n$, we have:

$$
p f p(\mathcal{D}, x)=\sum_{d_{i} \in \mathcal{D}} p f p\left(d_{i}, x\right)
$$

Table 2 shows an example of composable locality analysis. Three of the rows show the measured locality for each datum. The benefit of composable analysis is shown in the bottom four rows. The right-hand side shows the aggregate locality for accesses to two or more data items, which we can compute using Eq. 4. If we were to measure using the TP traces, also shown in the table, we would have obtained the same result as we do from calculation. 
Table 2. Example composition of single-datum locality (in upper table) to compute data-group locality (in lower table).

\begin{tabular}{|c|c|c|c|c|}
\hline \multirow{2}{*}{ Locality types } & \multirow{2}{*}{ TP traces } & \multicolumn{3}{|c|}{ Locality pfp $\left(d_{i}, x\right)$} \\
\cline { 2 - 5 } & & $x=1$ & $x=2$ & $x \geq 3$ \\
\hline \begin{tabular}{c} 
single-datum $\begin{array}{c}\text { focality } \\
\text { (measured) }\end{array}$ \\
\cline { 2 - 5 }
\end{tabular} & a__a__a__... & $1 / 3$ & $2 / 3$ & 1 \\
\cline { 2 - 5 } & _b_c_b__b_... & $1 / 3$ & $2 / 3$ & 1 \\
\hline \hline \multirow{2}{*}{$\begin{array}{c}\text { data group } \\
\text { locality } \\
\text { (computed) }\end{array}$} & ab_ab_ab_... & $1 / 3$ & $2 / 3$ & 1 \\
\cline { 2 - 5 } & _bc_bc_bc... & $2 / 3$ & $4 / 3$ & 2 \\
\cline { 2 - 5 } & a_ca_ca_c... & $2 / 3$ & $4 / 3$ & 2 \\
\cline { 2 - 5 } & abcabcabc... & 1 & 2 & 3 \\
\hline
\end{tabular}

\subsubsection{All-group Cache Performance}

Let $\mathcal{D}$ be the data group allocated to use the fully associative LRU cache of size $c$. The group miss ratio $\operatorname{mr}(\mathcal{D}, c)$ is computed by first obtaining the group footprint $p f p(\mathcal{D}, x)$ using Eq. 4 and then applying the HOTL conversion as follows

$$
m r(\mathcal{D}, c)=\left.\frac{\mathrm{d}}{\mathrm{d} x} p f p(\mathcal{D}, x)\right|_{p f p(\mathcal{D}, x)=c}
$$

Eq. 5 computes the group miss ratio from the group footprint. The derivative is taken when the group footprint equals to the cache size, i.e. $p f p(\mathcal{D}, x)=c$, which means that the cache is used by (and only by) data in $\mathcal{D}$.

Consider a trivial example. Let a be the only variable (group) stored in cache and its TP trace $a_{-} a_{-} a_{-} \ldots$. Using $p f p(a, x)$ from Table 2 and applying Eq. 5 for 0 -size cache, we see that the cache is read every third access, and it is all misses.

$$
m r(\mathrm{a}, 0)=\left.\frac{\mathrm{d}}{\mathrm{d} x} p f p(\mathrm{a}, x)\right|_{f p(x)=0}=p f p(\mathrm{a}, 1)-p f p(\mathrm{a}, 0)=\frac{1}{3}
$$

Combining composition (Eq. 4) and conversion (Eq. 5), we can measure the partial footprint for each array and compute the miss ratio of any data group in any size cache. The computed miss ratio has two theoretical properties.

Normalization Eq. 5 computes the miss ratio as the number of misses divided by $n$, the number of all data accesses (all of $\Omega$ ). A TP stream has placeholders. The traditional definition of the miss ratio should be the number of misses divided by the number of actual accesses (non-placeholders). The two definitions are different. To distinguish, we call them the normalized miss ratio and the actual miss ratio. For the example TP stream a__ a__ a__... at cache size 0 , the normalized miss ratio is $\frac{1}{3}$, and the actual miss ratio is $100 \%$.

Normalized miss ratios can be added directly (because of normalization, i.e. same denominator $n$ ), while actual miss ratios cannot. The equations of this paper always use normalized miss ratios.
Composition Invariance The group miss ratio of $\mathcal{D}=$ $\left\{d_{i}\right\}$ can be computed in two ways: directly from the group footprint or indirectly from the individual miss ratios. The direct solution is given before as Eq. 4 and Eq. 5. Eq. 4 computes the group footprint from individual footprints. The direct method applies the HOTL conversion on the left-hand side of Eq. 4. We can instead use the conversion on the right-hand side of Eq. 4.

In the indirect solution, we apply the HOTL conversion on the partial footprint of individual $d_{i}$ s and then add the miss ratios:

$$
\begin{aligned}
& m r\left(d_{i}, c\right)=\left.\frac{\mathrm{d}}{\mathrm{d} x} p f p\left(d_{i}, x\right)\right|_{p f p(\mathcal{D}, x)=c} \\
& m r(\mathcal{D}, c)=\sum_{d_{i} \in \mathcal{D}} m r\left(d_{i}, c\right)
\end{aligned}
$$

A useful feature of the indirect solution is Eq. 7, which shows the contribution of misses by each variable, $\operatorname{mr}\left(d_{i}, c\right)$ on the right-hand side, to the group miss ratio, $\operatorname{mr}(\mathcal{D}, c)$ on the left-hand side. In computing the $m r\left(d_{i}, c\right)$ in Eq. 6, the derivative is taken at $p f p(\mathcal{D}, x)=c$, which means that the cache is used by the whole $\mathcal{D}$, even though the miss ratio is computed only for $d_{i}$. This is the same condition used by the direct solution in Eq. 5. As a result composition correctness (formalized in Section 2.5.4), we can prove the following equality.

The two solutions, $m r(\mathcal{D}, c)$ from Eq. 5 and that from Eq. 7, are always equal for the same $c$. This equality shows composition invariance. As defined by $\mathrm{Hu}$ et al. [12], a composable model has composition invariance if the group miss ratio is the same whether it is computed from the composite locality or from adding together the miss ratio computed from the individual locality. Early composable models used reuse distance and footprint and had only one way to compute the group miss ratio [3, 20, 24]. Recent models have composition invariance, including AET [12] and HOTL based models [2, 25]. All previous work targeted composition of task locality. The partial footprint is the first to enable invariant composition of data locality.

\subsection{Mapped Footprint to Model Associativity}

This section presents the mapped footprint to model set associative cache and compares it with two previous models.

\subsubsection{Mapping the Footprint}

A set-associative cache of size $c$ is partitioned into $s$ cache sets $\mathcal{S}_{i}$, for $i=1, \ldots, s$. Each set has the same number of cache blocks, $\frac{c}{s}$, which is the associativity. The mapped footprint models two parameters of a set-associative cache: the number of sets $s$ and the cache size $c$ that is a multiple of $s$.

Address mapping is how each data block is mapped to a cache set based on its memory address. We assume that the 
mapping can be arbitrary but known to the analysis, and we also assume that a cache set is fully associative with LRU replacement.

First, we consider a single set count $s$. Following the idea of per-set reuse distance $[16,18]$, we measure the partial footprint for accesses to each cache set $\mathcal{S}_{i}$ as its mapped footprint, which is $p f p\left(\mathcal{S}_{i}, x\right)$. It is measured by first decomposing the execution trace into TP traces, one for each $\mathcal{S}_{i}$. The decomposition is the same as described in Section 2.2.1 and partial footprint defined in Section 2.2.2.

The miss ratio of the set-associative cache is computed as follows:

$$
\begin{aligned}
\text { sets } & =\left\{\mathcal{S}_{i}\right\}, i=1, \ldots, s \\
m r\left(\mathcal{S}_{i}, \frac{c}{s}\right) & =\left.\frac{\mathrm{d}}{\mathrm{d} x} p f p\left(\mathcal{S}_{i}, x\right)\right|_{p f p\left(\mathcal{S}_{i}, x\right)=\frac{c}{s}} \\
m r(c) & =\sum_{i=1}^{s} m r\left(\mathcal{S}_{i}, \frac{c}{s}\right)
\end{aligned}
$$

Eq. 8 computes the cache-set miss ratio by taking the derivative of a mapped footprint at cache associativity. Eq. 9 computes the miss ratio of the cache by adding the miss ratio of all sets. Simple addition is valid because these are normalized miss ratios (see Section 2.2.4).

Next, we consider multiple set counts. Assume the number of sets in a cache may be $s_{1}, s_{2}, \ldots$ Let $\hat{s}$ be their least common multiple, $\hat{s}=\operatorname{lcm}\left(s_{1}, s_{2}, \ldots\right)$. In this group of set counts, every $s_{k}$ divides $\hat{s}$, or $s_{k} \mid \hat{s}$. We measure the mapped footprint for every set $\mathcal{S}_{\hat{s}, i}$ when a cache has $\hat{s}$ sets and compute the mapped footprint for every set $\mathcal{S}_{s_{k}, i}$ when a cache has $s_{k}$ sets. To disambiguate, we have added the total number of sets as the first subscript of the set symbol.

The set of data blocks mapped to a $\mathcal{S}_{s_{k}, i}$ set is the disjoint union of the data blocks mapped to $\frac{\hat{s}}{s_{k}}$ instances of $\mathcal{S}_{\hat{s}, j}$ sets. We compute the mapped footprint of the larger set by adding the mapped footprint of the component sets:

$$
p f p\left(\mathcal{S}_{s_{k}, i}, x\right)=\sum_{\text {all js.t. } \mathcal{S}_{\hat{s}, j} \text { in }} p f p\left(\mathcal{S}_{s_{s_{k}}, i}, x\right)
$$

For example, assume that a cache is reconfigurable, and it has either 64 or 32 sets, namely, $s_{1}=64, s_{2}=32$. The least common multiple $\hat{s}$ is 64 . Given a program, we measure 64 mapped footprints, $p f p\left(\mathcal{S}_{64, i}, x\right)$ for $i=1, \ldots, 64$. To model the 32-set cache, we first compute their mapped footprints. Assuming linear mapping, i.e. for each $i=1, \ldots, 32$, we have $p f p\left(\mathcal{S}_{32, i}, x\right)=p f p\left(\mathcal{S}_{64, i}, x\right)+p f p\left(\mathcal{S}_{64, i+32}, x\right)$. This composition is an application of Eq. 10 and illustrated pictorially in Figure 1. The miss ratio is then computed by Eq. 8 for each set and Eq. 9 for the whole cache.

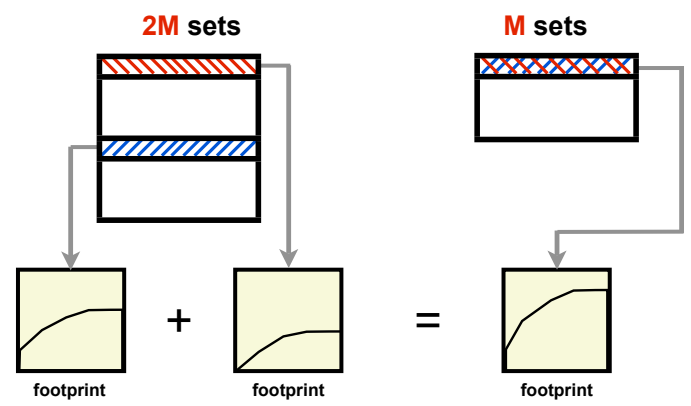

Figure 1. Two mapped footprints of the $2 M$-set cache are used to compute a mapped footprint of the $M$-set cache. The mapped footprints can then compute the miss ratio of any size cache with $M$ or $2 M$ sets.

The number of mapped footprints, $\hat{s}$, affects the memory cost of the analysis. In practice, we anticipate that the possible set counts, $s_{i}$, are multiples of each other. Then the number of the mapped footprints we need is the same as the largest set count.

We now have the complete technique to model any set count $s_{k}$ in Eq. 10 and any size $c$ in Eqs. 8, 9. The composition is the same for the mapped footprint in Eq. 10 as it is for the partial footprint in Eq. 4. A partial footprint measures the locality of a variable, while a mapped footprint the locality of a cache set. The former is non-uniformity in software, and the latter the heterogeneity in hardware. They are modeled by the same techniques: TP decomposition and footprint composition.

\subsubsection{Three Models of Associative Cache}

Smith gave the classic model in 1976 [19]. It assumes even mapping, i.e. each data item is to be found in any set equally likely, and the placement of one access has no dependence with other accesses. The Smith model is statistic. Each access is an independent, identically distributed random variable, equivalent to flipping an $s$-head coin, where $s$ is the number of sets in the cache. To account for the LRU replacement in each set, Smith used the reuse distance. The Smith model has been widely used and shown to be accurate for CPU caches [10, 14, 17].

There are two causes of cache conflicts: limited associativity and uneven mapping. The Smith model is a complete solution to the associativity problem but not the mapping problem. The mapped footprint solves both problems. The Smith assumption is the special case when the mapped footprint is identical across all sets.

Sen and Wood [18] and Nugteren et al. [16] created the first accurate solution, which measures the reuse distance in each cache set and then uses the per-set reuse distance to predict the miss ratio. Sen and Wood called it per-set locality [18]. Following Sen and Wood, we call this model per set reuse distance, or Set-RD in short. 
Set-RD is accurate in solving both the associativity and mapping problems but for a single set count. The mapped footprint extends the idea of Set-RD but uses footprint instead of reuse distance. As a result, it supports multiple set counts (Eq. 10).

Table 3 shows the three models in terms of the generality and accuracy. A general model can predict the miss ratio for a cache of any associativity and any set count. Note that the accuracy of mapped footprint refers to the composition of footprints (Section 2.5.4).

Table 3. Models of Associative Cache

\begin{tabular}{|c|c|c|c|c|}
\hline \hline \multicolumn{2}{|c|}{} & Smith [19] & Set-RD [16, 18] & MFP \\
\hline \hline \multirow{2}{*}{ generality } & associativity & all & all & all \\
\cline { 2 - 5 } & set count & all & one & multiple \\
\hline \multirow{2}{*}{ accuracy } & data mapping & uniform & arbitrary & arbitrary \\
\cline { 2 - 5 } & $\mathbf{m r} / \mathbf{r d} / \mathbf{f p}$ & approx. & precise & precise \\
\hline
\end{tabular}

\subsection{Dual-grained Footprint to Model Sub-block Cache}

In this section, we model another caching technique called sub-block cache or sector cache, which divides the cache block into sectors, fetches data in sector granularity, but manages data in cache-line granularity. We call cache-line granularity the block granularity. This design is proposed by Cox et al. [6]. We analyze the performance of such cache through a new model called dual-grained footprint.

\subsubsection{Dual-grained Footprint}

Consider two granularities, $\mathcal{G}_{s c t r}, \mathcal{G}_{b l k}$, measured in bytes, e.g. $\mathcal{G}_{\text {sctr }}=32, \mathcal{G}_{b l k}=128$. We measure dual-grained footprints, one for each granularity, denote them $\operatorname{gfp}\left(\mathcal{G}_{\text {sctr }}, x\right)$ and $g f p\left(\mathcal{G}_{b l k}, x\right)$, and call them sector footprint and block footprint respectively.

The miss ratio of the sector cache is computed as follows:

$$
m r(c)=\left.\frac{\mathrm{d}}{\mathrm{d} x} g f p\left(\mathcal{G}_{s c t r}, x\right)\right|_{g f p\left(\mathcal{G}_{b l k}, x\right)=c}
$$

In Eq. 11, the derivative is computed by the sector footprint, while the point at which the derivative is taken is determined by the block footprint. It is dual grained and uses two units of measure: the miss frequency is measured in the unit of sectors (since each miss is a sector), while the cache size is measured in the unit of cache blocks. The dual grained calculation models the fact that the sector cache operates in different granularities.

Consider the example in Table 4. For simplicity, let's assume fully associative sector cache, where each sector stores an array element, and each block has two sectors, i.e. $\mathcal{G}_{\text {sctr }}=$ $1, G_{b l k}=2$.
Table 4. Example Analysis of Sector Cache

(a) Accesses in sector and block granularity

access trace: $a[1] \quad a[2] \quad b[1] \ldots$

block trace: $a[1: 2] a[1: 2] b[1: 2] \ldots$

(b) Footprints in two granularities

\begin{tabular}{c|c|c|c} 
time scale $x$ & 0 & 1 & 2 \\
\hline$g f p\left(\mathcal{G}_{s c t r}, x\right)$ & 0 & 1 & 2 \\
$g f p\left(\mathcal{G}_{b l k}, x\right)$ & 0 & 1 & $\frac{5}{3}$
\end{tabular}

(c) Miss ratios by single- and dual-granularity footprints

\begin{tabular}{c|c|c} 
cache size c & 0 & 1 \\
\hline dual granularity (Eq. 11) & $100 \%$ & $100 \%$ \\
block granularity (Eq. 2) & $100 \%$ & $67 \%$
\end{tabular}

Let the trace be a repeat of 3 accesses, $a[1] a[2] b[1]$ $\ldots$, and the cache capacity be 1 block. From dual-granularity footprint, Eq. 11 computes the miss ratio $100 \%$, which means 3 sectors every 3 accesses. From single (block) granularity, HOTL (Eq. 2) computes 67\%, which means 2 blocks (4 sectors) every 3 accesses. The latter is wrong. It fails to show the benefit of sector cache, i.e. not loading unused data.

Just the sector granularity can also be wrong. It fails when some sector in a cache block is unused. Let the trace be a repeat of 6 accesses to 4 array elements, $a[1] a[2] b[1]$ $\mathrm{a}[1] \mathrm{a}[2] \mathrm{c}[1] \ldots$, and the cache size be 2 blocks. Using HOTL with the sector footprint, we take the derivative at $x=6$ because $g f p\left(\mathcal{G}_{s c t r}, 6\right)=4$ and incorrectly predict all accesses as hits. Using dual-granularity footprint, we take the derivative at $x=3$, since $g f p\left(\mathcal{G}_{b l k}, 3\right)=2$, and predict $a[1] a[2]$ as hits and $b[1] c[1]$ as misses, which is correct.

The example calculations used two unit measures. The misses were counted in the unit of sectors, and the cache size was measured in the unit of blocks. These are independent units yet used in the same equation. In fact, any model of sector cache must account for different unit values, for which dual-granularity footprint is a nature fit.

\subsection{Inter-Model Locality Composition}

In the two preceding sections, we have presented solutions to model non-uniform locality and cache heterogeneity. In this section, we bring the combined power of these solutions to bear on modeling the joint effect of set associative cache with sub-block placement policy, we named such cache as set associative sub-block cache.

\subsubsection{Non-uniform Locality in Set associative Cache}

Assume the cache has $s$ sets $\left\{\mathcal{S}_{i}\right\}, i=1, \ldots, s$. We measure the partial footprint per variable-set pair $\left\langle\mathcal{S}_{i}, d_{j}\right\rangle$, i.e. $p f p\left(\left\langle\mathcal{S}_{i}, d_{j}\right\rangle, x\right)$ of the TP trace with only accesses to the part of array $d_{j}$ that is mapped to cache set $\mathcal{S}_{i}$. For any array 
group $\mathcal{D}=\left\{d_{j}\right\}$, we compute the mapped group footprint per cache set $p f p\left(\left\langle\mathcal{S}_{i}, \mathcal{D}\right\rangle, x\right)$ and the cache miss ratio as follows:

$$
\begin{aligned}
p f p\left(\left\langle\mathcal{S}_{i}, \mathcal{D}\right\rangle, x\right) & =\sum_{d_{i} \in \mathcal{D}} p f p\left(\left\langle\mathcal{S}_{i}, d_{i}\right\rangle, x\right) \\
m r\left(\mathcal{S}_{i}, \frac{c}{s}\right) & =\left.\frac{\mathrm{d}}{\mathrm{d} x} p f p\left(\left\langle\mathcal{S}_{i}, \mathcal{D}\right\rangle, x\right)\right|_{p f p\left(\left\langle\mathcal{S}_{i}, \mathcal{D}\right\rangle, x\right)=\frac{c}{s}} \\
m r(c) & =\sum_{i=1}^{s} m r\left(\mathcal{S}_{i}, \frac{c}{s}\right)
\end{aligned}
$$

The last equation shows the miss ratio of all variables in $\mathcal{D}$ in the set-associative cache with $s$ sets and $c$ blocks. The calculation applies to any variable group in software and set-associative cache of any size and associativity and (not shown in the equations) any number of sets.

\subsubsection{Non-uniform Locality in Sub-block Cache}

We have shown the use of partial footprint of each variable $p f p\left(d_{i}, x\right)$ to model non-uniform locality and dual-grained footprints $g f p\left(\mathcal{G}_{s t r}, x\right), g f p\left(\mathcal{G}_{b l k}, x\right)$ to model sector cache. We combine the two concepts and use dual-grained partial footprint per variable, $g p f p\left(\mathcal{G}_{s c t r}, d_{i}, x\right)$ and $g p f p\left(\mathcal{G}_{b l k}, d_{i}, x\right)$, to model the sector cache performance for the group $\mathcal{D}=\left\{d_{i}\right\}$ as follows:

$$
\begin{aligned}
g f p\left(\mathcal{G}_{s c t r}, x\right) & =\sum_{d_{i} \in \mathcal{D}} g p f p\left(\mathcal{G}_{s c t r}, d_{i}, x\right) \\
g f p\left(\mathcal{G}_{b l k}, x\right) & =\sum_{d_{i} \in \mathcal{D}} g p f p\left(\mathcal{G}_{b l k}, d_{i}, x\right) \\
m r(c) & =\left.\frac{\mathrm{d}}{\mathrm{d} x} \operatorname{gfp}\left(\mathcal{G}_{s c t r}, x\right)\right|_{g f p\left(\mathcal{G}_{b l k}, x\right)=c}
\end{aligned}
$$

The last equation shows the miss ratio of all variables in $\mathcal{D}$ in the sector cache of size $c$. The calculation applies to any variable group in software and sector cache of any size.

\subsubsection{Locality in Sub-block Set associative Cache}

In this section, we combine the mapped footprint and dualgrained footprint to model the sub-block set-associative cache. Given the set associate cache with size $c$ and partition sets $\left\{\mathcal{S}_{i}\right\}, i=1, \ldots, s$. Define $\hat{s}$ to be the least common multiple for all set size $s_{1}, s_{2}, \ldots$, we can derive the cache performance through the dual-grained footprint per cache set $g f p\left(\mathcal{G}_{s c t r}, \mathcal{S}_{s_{k}, i}, x\right)$ and $g f p\left(\mathcal{G}_{b l k}, \mathcal{S}_{s_{k}, i}, x\right)$ as follows:

$$
\begin{aligned}
g f p\left(\mathcal{G}_{s c t r}, \mathcal{S}_{s_{k}, i}, x\right) & =\sum_{\text {all } j \text { s.t. }} \sum_{\mathcal{S}_{\hat{s}, j} \text { in }} \operatorname{gfp} \mathcal{S}_{s_{k}, i} \operatorname{gl}\left(\mathcal{G}_{s c t r}, \mathcal{S}_{\hat{s}, j}, x\right) \\
g f p\left(\mathcal{G}_{b l k}, \mathcal{S}_{s_{k}, i}, x\right) & =\sum_{\text {all } j \text { s.t. }} g f p\left(\mathcal{S}_{\hat{s}, j} \text { in } \mathcal{S}_{s_{k}, i}, \mathcal{S}_{\hat{s}, j}, x\right) \\
m r(c) & =\left.\frac{\mathrm{d}}{\mathrm{d} x} g f p\left(\mathcal{G}_{s c t r}, \mathcal{S}_{s_{k}, i}, x\right)\right|_{g f p\left(\mathcal{G}_{b l k}, \mathcal{S}_{s_{k}, i}, x\right)=c}
\end{aligned}
$$

The last equation shows the miss ratio of all the cache sets in the sub-block set associative cache of size $c$.

\subsubsection{Correctness}

We formalize the correctness of partial footprint composition. Let $s_{i}$ for $i=1, \ldots, k$ be any set of time-preserving (TP) streams of $k$ (non-overlapping) data variables, and $p f p\left(s_{i}, x\right)$ for $i=1, \ldots, k$ be the partial footprint of $s_{i}$ as defined in Eq. 3. We have the following theorem.

Composition Theorem. Let $\left\{s_{i}\right\}(1 \leq k \leq k)$ be a set of $k$ TP traces that do not access the same data, and $\oplus_{i=1}^{k} s_{i}$ be the combined TP trace. The footprint of the combined trace is the sum of the footprint of its individual traces, i.e. pfp $\left(\oplus_{i=1}^{k} s_{i}, x\right)=$ $\sum_{i=1}^{k} p f p\left(s_{i}, x\right)$.

Proof. In every time window $(j, x)$, let omega $\left(\oplus_{i=1}^{k} s_{i}, j, x\right)$ be the working-set size of all $d_{i}$ s. Since the partial working set is disjoint for different $d_{i} \mathrm{~s}$, the partial working-set size is additive across all $d_{i}$ s. Therefore, omega $\left(\oplus_{i=1}^{k} s_{i}, j, x\right)=$ $\sum_{i=1}^{k} \omega\left(s_{i}, j, x\right)$. Hence, the average of omega $\left(\oplus_{i=1}^{k} s_{i}, j, x\right)$ is the sum of the average of $\omega\left(s_{i}, j, x\right)$. In other words, the footprint of the interleaved stream is the sum of the partial footprint of all $d_{i} \mathrm{~s}$.

The theorem proves the accuracy of composition in the model of non-uniform locality Eqs. 4 and 5, of cache sets Eq. 10, and their uses in the combined analysis earlier in this section. Since the proof does not assume any specific interleaving, the correctness holds for arbitrary interleaving patterns for a set of variables $\mathcal{D}=\left\{d_{i}\right\}$ or cache sets $\left\{\mathcal{S}_{i}\right\}$, for examples:

- A variable or a cache set may be accessed any number of times. One may be accessed 100 times more often than another.

- The accesses of a variable or a cache set may be arbitrarily distributed in time. The rate of access may vary for the same variable, and it may happen that most accesses are clustered in one time period.

- Two variables or cache sets may not be accessed at the same time, e.g., all accesses in one variable may predate all accesses of the other variable.

Miss Ratio Conversion The HOTL conversion from footprint to miss ratio by Eq. 2 is correct if the reuse window 
hypothesis holds, which states: the footprint in all reuse windows has the same distribution as the footprint in all windows, for every window length [25]. Drudi gave a contrived example, where the footprint predicts incorrectly [9]. The correct solution is reuse distance, as used in $[16,18]$. The accuracy of HOTL conversion has been tested extensively for set-associative CPU caches $[12,25]$ and fully associative storage caches $[9,12,22]$.

\section{Evaluation}

We evaluate our models described above using 13 benchmarks from SHOC [7] and CUDA Code Samples [15]. Table 5 gives the brief description for each benchmark.

Table 5. Benchmarks for evaluation

\begin{tabular}{|c|c|}
\hline Benchmarks & Description \\
\hline \hline BFS & graph algorithm \\
\hline FFT & numerical algorithm \\
\hline Scan & parallel prefix sum \\
\hline Reduction & reduce routine \\
\hline Sort & sorting \\
\hline Triad & vector addition \\
\hline Stencil2D & structured grid \\
\hline Trans & matrix transpose \\
\hline MM & matrix multiply \\
\hline MD & N-Body simulation \\
\hline SpMV & matrix-vector multiply \\
\hline CFD & unstructured grid \\
\hline ParticleFilter & structured grid \\
\hline
\end{tabular}

We compare our cache models with three existing techniques listed in Table 6. The memory traces [4] are collected from a Tesla M2075 general-purpose graphics processor, a Fermi device with Compute Capability 2.0. We run all four techniques (including our cache models) on a simulator which simulates two caches, a 48KB, 6-way L1 cache and a $12 \mathrm{~KB}, 8$-way sector cache, both of which are selected to match the configuration on Tesla M2075.

Table 6. Three alternative cache models

\begin{tabular}{|c|l|}
\hline Models & \multicolumn{1}{c|}{ Features } \\
\hline \hline Baseline & simulated set associative LRU cache and sector cache. \\
\hline Set-RD [16] & $\begin{array}{l}\text { a fixed number of cache sets and one reuse distance } \\
\text { histogram per cache set. }\end{array}$ \\
\hline PORPLE [4] & $\begin{array}{l}\text { 1. reuse distance of whole trace, no set associativity. } \\
\text { 2. assume arrays partition cache space equally. }\end{array}$ \\
\hline
\end{tabular}

Modeling Accuracy To measure the accuracy of our models, we first identify the arrays in a program that can be placed in different memories and have significant performance impact. In general, there are three ways to place an array: 1) Place the array in L1 cache. 2) Place the array in sector cache if it is read only. 3) Not cache the array.

In our benchmark suite, 10 out of 13 programs have 2 to 7 arrays, which means in maximum $3^{2}=9$ to $3^{7}=2187$ possible placements. We enumerate the miss ratio results of for all possible array placements and present the result in Table 7. Each row in that table is a measurement and may represent multiple placement choices. For example, the second row of Sort has Array I in L1 and Array II in the sector cache. It also implies the cache performance for two more placements: when Array I is in L1 and Array II not cached, and when Array I is not cached and Array II in sector cache.

For each placement, the table shows one simulated miss ratios: Baseline, and three predictions: Set-RD, PORPLE and Footprint. The simulation runs the memory trace once for each placement. The prediction profiles the memory trace once for all placements. Set-RD is precise. It gives the same results as the Baseline simulation, so the table shows the two in a single column.

Table 7 shows that our cache models are fairly accurate compared to simulation. Most predictions are either identical to the simulation result or differ by a negligible amount. The largest error is $49 \%$ prediction v.s. $42 \%$ simulation in one of the four placements of $M M$. In comparison, PORPLE does not model data non-uniformity. When a set of arrays share the cache, it assumes equal cache occupancy.

For L1 cache, PORPLE is mostly accurate but has large errors occasionally, e.g. by $15 \%$ in the first case of $M D, 5 \%$ to $23 \%$ in four cases in $S p M V$, a factor of 2 in two cases in ParticleFilter and one case in CFD. Array III of $S p M V$ is accessed irregularly and causes contention in L1 that disrupts the dynamic balance. PORPLE prediction is almost always wrong. The relative error is as high as $83 \%$. These results show that there is significant data non-uniformity.

For sector cache, as Table 7 shows, Footprint is as accurate for the sector cache as it is for L1. The highest error is $36 \%$ prediction v.s. $29 \%$ simulation in one of the 11 placements of SpMV. In contrast, the prediction errors of PORPLE are relatively greater in the sector cache, e.g. $20 \%$ in all 3 cases of $M M$, as it employs a crude approach which models the sector cache by a fully associative, sector-granularity, LRU cache. The larger errors of PORPLE indicate that the sector cache differs from L1, and our model captures this difference.

Modeling Cost Table 8 shows the total cost of modeling for each test program, normalized by the time of a single cache simulation run. Most of the cost of our Footprint model is profiling for reuse time. The rest is converting it to footprints and miss ratios. The time for predicting for one placement is negligible, less than $0.1 \%$ of profiling. Therefore prediction time is not included in the table.

PORPLE and Set-RD profile a program trace to measure the reuse distance. The implementations follow the algorithms by Kim et al. [13] and Almasi et al. [1] respectively. Both 
Table 7. Cache miss ratios by different array placements. Each array has at most three placing options: L1, sector cache or not cached. The column array placement in cache shows the placement for at most four arrays, where "L1" means L1 data cache, configured 48KB, "Sec" means the sector cache, and "-" means either no such array or that the array is not cached in either L1 or Sec. For some benchmarks, not all placements are shown for brevity. For each placement, the table compares four methods: one simulated miss ratios, Baseline; and three predictions, Set-RD, Footprint and PORPLE. The simulation runs on a program trace once for each placement. The prediction runs on a program trace once for all placements.

\begin{tabular}{|c|c|c|c|c|c|c|c|c|c|c|c|}
\hline & \multirow{3}{*}{$\begin{array}{l}\text { Number of } \\
\text { placements }\end{array}$} & \multicolumn{4}{|c|}{ Array placement in caches } & \multicolumn{3}{|c|}{ L1 cache miss ratio (\%) } & \multicolumn{3}{|c|}{ Sector cache miss ratio (\%) } \\
\hline & & \multirow{2}{*}{ I } & \multirow{2}{*}{ II } & \multirow{2}{*}{ III } & \multirow{2}{*}{ IV } & Simulation & \multicolumn{2}{|c|}{ Model } & \multirow{2}{*}{$\begin{array}{c}\text { Simulation } \\
\text { Baseline }\end{array}$} & \multicolumn{2}{|c|}{ Model } \\
\hline & & & & & & \begin{tabular}{l|l} 
Baseline & Set-RD \\
\end{tabular} & Footprint & PORPLE & & Footprint & PORPLE \\
\hline \multirow{7}{*}{ BFS } & \multirow{7}{*}{$\begin{array}{c}\mathbf{8} \\
\text { (3 arrays) }\end{array}$} & L1 & L1 & L1 & - & 1.67 & 1.67 & 1.67 & - & - & - \\
\hline & & L1 & Sec & L1 & - & 2.80 & 2.80 & 2.80 & 1.16 & 1.23 & 0.53 \\
\hline & & L1 & Sec & Sec & - & 3.20 & 3.30 & 3.20 & 1.70 & 1.75 & 1.02 \\
\hline & & - & $\mathrm{L} 1$ & L1 & - & 1.43 & 1.43 & 1.43 & - & - & - \\
\hline & & $\mathrm{L} 1$ & $\mathrm{~L} 1$ & $\mathrm{Sec}$ & - & 1.98 & 1.98 & 1.98 & 3.36 & 3.58 & 13.02 \\
\hline & & - & $\mathrm{Sec}$ & $\mathrm{L} 1$ & - & 3.36 & 3.36 & 3.36 & 1.16 & 1.23 & 0.53 \\
\hline & & - & L1 & $\mathrm{Sec}$ & - & 1.16 & 1.16 & 1.10 & 3.36 & 3.58 & 13.02 \\
\hline & & L1 & - & - & - & 100.00 & 100.00 & 100.00 & - & - & - \\
\hline Scan & 2 & $\mathrm{Sec}$ & - & - & - & - & - & - & 100.00 & 100.00 & 100.00 \\
\hline & & L1 & - & - & - & 99.70 & 100.00 & 100.00 & - & - & - \\
\hline Reduction & 2 & $\mathrm{Sec}$ & - & - & - & - & - & - & 49.95 & 49.95 & 50.00 \\
\hline & & L1 & L1 & - & - & 100.00 & 100.00 & 100.00 & - & - & - \\
\hline & 4 & $\mathrm{Sec}$ & $\mathrm{L} 1$ & - & - & 100.00 & 100.00 & 100.00 & 100.00 & 100.00 & 100.00 \\
\hline Sort & (2 arrays) & $\mathrm{Sec}$ & $\mathrm{Sec}$ & - & - & - & - & - & 99.90 & 99.90 & 100.00 \\
\hline & & L1 & $\mathrm{Sec}$ & - & - & 100.00 & 100.00 & 100.00 & 99.80 & 99.80 & 100.00 \\
\hline & & L1 & - & - & - & 38.09 & 38.09 & 38.62 & - & - & - \\
\hline Stencil2D & 2 & $\mathrm{Sec}$ & - & - & - & - & - & - & 46.79 & 46.79 & 33.56 \\
\hline Trons & 2 & L1 & - & - & - & 75.00 & 75.00 & 75.00 & - & - & - \\
\hline Irans & 2 & $\mathrm{Sec}$ & - & - & - & - & - & - & 49.95 & 49.95 & 50.00 \\
\hline & & L1 & L1 & - & - & 0.38 & 0.31 & 0.58 & - & - & - \\
\hline ParticleFilter & 3 & $\mathrm{~L} 1$ & - & - & - & 0.88 & 0.83 & 1.59 & - & - & - \\
\hline & & - & L1 & - & - & 0.02 & 0.02 & 0.02 & - & - & - \\
\hline & & L1 & $\mathrm{Sec}$ & $\mathrm{Sec}$ & $\mathrm{Sec}$ & 6.66 & 6.60 & 6.66 & 45.32 & 49.10 & 49.93 \\
\hline & & Sec & $\mathrm{L} 1$ & Sec & Sec & 50.18 & 50.18 & 50.18 & 40.38 & 45.34 & 53.80 \\
\hline & & Sec & Sec & L1 & Sec & 6.31 & 6.31 & 6.31 & 35.67 & 35.76 & 35.60 \\
\hline & & Sec & Sec & Sec & L1 & 51.20 & 51.20 & 50.18 & 40.52 & 45.34 & 53.82 \\
\hline & & Sec & L1 & L1 & L1 & 22.13 & 20.81 & 24.62 & 1.56 & 1.95 & 1.66 \\
\hline SpMV & 16 & L1 & Sec & L1 & L1 & 14.80 & 14.08 & 19.80 & 35.74 & 35.76 & 36.08 \\
\hline & (4 arrays) & L1 & L1 & Sec & L1 & 49.71 & 49.71 & 49.71 & 29.45 & 36.33 & 38.74 \\
\hline & & L1 & L1 & L1 & $\mathrm{Sec}$ & 14.66 & 14.06 & 19.77 & 35.76 & 35.77 & 36.05 \\
\hline & & L1 & $\mathrm{L} 1$ & L1 & L1 & 22.09 & 20.77 & 43.22 & - & - & - \\
\hline & & Sec & Sec & L1 & L1 & 14.83 & 14.11 & 19.84 & 35.16 & 34.79 & 35.15 \\
\hline & & Sec & Sec & Sec & $\mathrm{Sec}$ & - & - & - & 45.05 & 48.77 & 59.66 \\
\hline FFT & 2 & L1 & - & - & - & 57.18 & 56.25 & 57.81 & - & - & - \\
\hline FFI & 2 & $\mathrm{Sec}$ & - & - & - & - & - & - & 72.98 & 73.68 & 73.43 \\
\hline & & L1 & L1 & - & - & 98.46 & 100.00 & 100.00 & - & - & - \\
\hline Triad & 4 & $\mathrm{Sec}$ & $\mathrm{L} 1$ & - & - & 96.96 & 100.00 & 100.00 & 50.00 & 50.00 & 50.00 \\
\hline HITa & 4 & Sec & Sec & - & - & - & - & - & 49.90 & 49.90 & 50.00 \\
\hline & & L1 & $\mathrm{Sec}$ & - & - & 100.00 & 100.00 & 100.00 & 49.80 & 49.80 & 50.00 \\
\hline & & L1 & L1 & - & - & 78.62 & 76.47 & 91.30 & - & - & - \\
\hline MD & & $\mathrm{Sec}$ & $\mathrm{L} 1$ & - & - & 100.00 & 100.00 & 100.00 & 96.81 & 96.89 & 94.38 \\
\hline NIO & 4 & $\mathrm{~L} 1$ & $\mathrm{Sec}$ & - & - & 77.14 & 75.00 & 82.83 & 50.00 & 50.00 & 50.00 \\
\hline & & $\mathrm{Sec}$ & $\mathrm{Sec}$ & - & - & - & - & - & 87.47 & 87.52 & 87.17 \\
\hline & & L1 & L1 & L1 & L1 & 37.69 & 31.40 & 57.50 & - & - & - \\
\hline & & $\mathrm{Sec}$ & $\mathrm{L} 1$ & $\mathrm{~L} 1$ & L1 & 26.74 & 20.54 & 7.03 & 59.90 & 59.23 & 53.15 \\
\hline CFD & (7 arrays) & $\mathrm{Sec}$ & $\mathrm{Sec}$ & $\mathrm{L} 1$ & L1 & 16.37 & 15.65 & 7.03 & 80.09 & 78.67 & 75.86 \\
\hline & & $\mathrm{Sec}$ & $\mathrm{L} 1$ & $\mathrm{Sec}$ & $\mathrm{Sec}$ & 7.03 & 7.03 & 7.03 & 80.19 & 79.32 & 75.34 \\
\hline & & L1 & L1 & - & - & 57.82 & 54.78 & 74.89 & - & - & - \\
\hline & & $\mathrm{L} 1$ & $\mathrm{Sec}$ & - & - & 50.11 & 50.11 & 50.10 & 29.64 & 29.64 & 50.00 \\
\hline MM & 4 & $\mathrm{Sec}$ & $\mathrm{L} 1$ & - & - & 41.78 & 48.68 & 3.42 & 30.97 & 30.97 & 50.00 \\
\hline & & $\mathrm{Sec}$ & $\mathrm{Sec}$ & - & - & - & - & - & 30.29 & 30.29 & 50.00 \\
\hline
\end{tabular}


Table 8. Time cost of different techniques, measured by the total time normalized to the time it takes to simulate the program for a single data placement.

\begin{tabular}{|c|c|c|c|c|}
\hline & \multicolumn{4}{|c|}{ Normalized cost } \\
\hline benchmarks & Baseline & Footprint & PORPLE & Set-RD \\
\hline \hline BFS & 8.0 & 0.4 & 0.1 & 21 \\
\hline FFT & 2.0 & 1.5 & 6.8 & 350 \\
\hline Scan & 2.0 & 1.1 & 1.3 & 54 \\
\hline Reduction & 2.0 & 0.8 & 0.7 & 118 \\
\hline Sort & 4.0 & 1.9 & 0.8 & 196 \\
\hline Triad & 4.0 & 2.2 & 0.2 & 240 \\
\hline Stencil2D & 2.0 & 0.7 & 0.5 & 78 \\
\hline Trans & 2.0 & 1.4 & 0.5 & 54 \\
\hline MM & 4.0 & 0.3 & 6.7 & 344 \\
\hline MD & 4.0 & 1.1 & 39 & 364 \\
\hline SpMV & 16.0 & 0.8 & 4.0 & 1072 \\
\hline CFD & 128.0 & 4.2 & 0.6 & 3840 \\
\hline ParticleFilter & 3.0 & 0.2 & 0.6 & 90 \\
\hline \hline mean & 14 & 1.6 & 4.7 & 524 \\
\hline
\end{tabular}

implementations use stack processing ${ }^{2}$. Stack processing has a high space overhead and greater asymptotic complexity. The cost increases dramatically for large traces. PORPLE is fast in most cases, but for one program it has 39 times slowdown due to the program's large memory footprint. In contrast, footprint analysis is constant time per trace element and has linear time complexity. Footprint based models have better scalability than reuse distance based models.

In short, Set-RD is accurate and predicts the miss ratio for all cache associativity. PORPLE is 111 times faster but does not model set associativity, which caused prediction errors in many cases. Our model is 3 times faster than PORPLE. The lower overhead (327 times faster than Set-RD) and higher accuracy (4.3 times lower error than PORPLE) show the benefit of our model.

\section{Summary}

In this paper, we have presented the first composable analysis to model the sub-block set associative cache. The techniques include time-preserving trace decomposition and partial footprints to model data placement, mapped footprint to model set associativity, and dual granularity footprint to model cache heterogeneity. We show that these models are composable to model the combined effect and the correctness, i.e. the composition theorem. The evaluation on 13 GPU benchmarks shows that the composable model takes a fraction of the time of a single cache simulation to predict the cache performance for all data placements, and the prediction is $99.2 \%$ accurate. Its average error is less than $25 \%$ of PORPLE, the previous composable technique.

\footnotetext{
${ }^{2}$ Asymptotically more efficient reuse distance measurements exist but we retain the original design of these two systems.
}

\section{Acknowledgments}

The authors would like to thank Bruce Jacob for shepherding the paper, and the comments and suggestions of the anonymous reviewers. This work was supported by the National Science Foundation (Contract No. CCF-1717877, CCF1629376, CCF-1455404, CCF-1525609, CNS-1717425, CCF1703487), DOE Early Career Award (DE-SC0013700) and IBM CAS Faculty Fellowship. Any opinions, findings, and conclusions or recommendations expressed in this material are those of the authors and do not necessarily reflect the views of DOE or NSF.

\section{References}

[1] George Almasi, Calin Cascaval, and David A. Padua. 2002. Calculating Stack Distances Efficiently. In Proceedings of the ACM SIGPLAN Workshop on Memory System Performance. Berlin, Germany, 37-43.

[2] Jacob Brock, Chencheng Ye, Chen Ding, Yechen Li, Xiaolin Wang, and Yingwei Luo. 2015. Optimal Cache Partition-Sharing. In Proceedings of ICPP.

[3] Dhruba Chandra, Fei Guo, Seongbeom Kim, and Yan Solihin. 2005. Predicting Inter-Thread Cache Contention on a Chip Multi-Processor Architecture. In Proceedings of HPCA. 340-351.

[4] Guoyang Chen, Bo Wu, Dong Li, and Xipeng Shen. 2014. PORPLE: An Extensible Optimizer for Portable Data Placement on GPU. In Proceedings of MICRO.

[5] Umang Choudhary, Pratik Phadke, Vasundhara Puttagunta, and Supreeth Udayashankar. [n. d.]. Analysis of Sub-block Placement and Victim Caching Techniques.

[6] Michael Cox, Narendra Bhandari, and Michael Shantz. 1998. Multilevel Texture Caching for 3D Graphics Hardware. In Proceedings of ISCA.

[7] Antony Danalis, G. Marin, C. McCurdy, J. S. Meredith, P. C. Roth, K. Spafford, V. Tipparaju, and J. S. Vetter. 2010. The Scalable Heterogeneous Computing (SHOC) Benchmark Suite. In GPGPU.

[8] Peter J. Denning. 1968. The working set model for program behaviour. Commun. ACM 11, 5 (1968), 323-333.

[9] Zachary Drudi. 2014. A Streaming Algorithms Approach to Approximating Hit Rate Curves. Technical Report MS Thesis. The University Of British Columbia.

[10] Mark D. Hill and Alan Jay Smith. 1989. Evaluating associativity in CPU caches. IEEE Trans. Comput. 38, 12 (1989), 1612-1630.

[11] Xiameng Hu, Xiaolin Wang, Yechen Li, Lan Zhou, Yingwei Luo, Chen Ding, Song Jiang, and Zhenlin Wang. 2015. LAMA: Optimized Localityaware Memory Allocation for Key-value Cache. In Proceedings of USENIX ATC.

[12] Xiameng Hu, Xiaolin Wang, Lan Zhou, Yingwei Luo, Chen Ding, and Zhenlin Wang. 2016. Kinetic Modeling of Data Eviction in Cache. In Proceedings of USENIX ATC. 351-364. https://www.usenix.org/ conference/atc16/technical-sessions/presentation/hu

[13] Yul H. Kim, Mark D. Hill, and David A. Wood. 1991. Implementing Stack Simulation for Highly-Associative Memories. In Proceedings of SIGMETRICS. 212-213.

[14] G. Marin and J. Mellor-Crummey. 2005. Scalable Cross-Architecture Predictions of Memory Hierarchy Response for Scientific Applications. In Proceedings of the Symposium of the Las Alamos Computer Science Institute. Sante Fe, New Mexico.

[15] NIVDIA. 2016. NIVDIA CUDA Code Samples. https://developer.nvidia. com/cuda-code-samples.

[16] Cedric Nugteren, Gert-Jan van den Braak, Henk Corporaal, and Henri E. Bal. 2014. A detailed GPU cache model based on reuse distance theory. In Proceedings of HPCA. 
[17] Apan Qasem and Ken Kennedy. 2005. A Cache-Conscious Profitability Model for Empirical Tuning of Loop Fusion. In Proceedings of the LCPC Workshop. 106-120. https://doi.org/10.1007/978-3-540-69330-7_8

[18] Rathijit Sen and David A. Wood. 2013. Reuse-based online models for caches. In Proceedings of SIGMETRICS. 279-292.

[19] A. J. Smith. 1976. On the Effectiveness of Set Associative Page Mapping and Its Applications in Main Memory Management. In Proceedings of ICSE.

[20] G. Edward Suh, Srinivas Devadas, and Larry Rudolph. 2001. Analytical cache models with applications to cache partitioning.. In Proceedings of ICS. 1-12.

[21] Wang et al. 2015. Optimal Program Symbiosis in Shared Cache. In Proceedings of CCGrid.

[22] Jake Wires, Stephen Ingram, Zachary Drudi, Nicholas JA Harvey, Andrew Warfield, and Coho Data. 2014. Characterizing storage workloads with counter stacks. In Proceedings of OSDI. USENIX Association, 335349.

[23] Meng-Ju Wu, Minshu Zhao, and Donald Yeung. 2013. Studying multicore processor scaling via reuse distance analysis. In Proceedings of ISCA. 499-510.

[24] Xiaoya Xiang, Bin Bao, Chen Ding, and Yaoqing Gao. 2011. Linear-time Modeling of Program Working Set in Shared Cache. In Proceedings of PACT. 350-360

[25] Xiaoya Xiang, Chen Ding, Hao Luo, and Bin Bao. 2013. HOTL: a higher order theory of locality. In Proceedings of ASPLOS. 343-356. 